\title{
Computing Robin Problem on Unbounded Simply Connected Domain via an Integral Equation with the Generalized Neumann Kernel
}

\author{
Shwan H. H. Al-Shatri \\ Science Department \\ Institute of Training and Educational \\ Development in Sualiamni-Iraq \\ kakshko0@gmail.com
}

\author{
Munira Ismail. \\ Department of Mathematical Sciences \\ Faculty of Science \\ Universiti Teknologi Malaysia, Malaysia \\ muniraismail@utm.my
}

\author{
Karzan Wakil \\ Sualimani Polytechnic University-Iraq \\ University of Human Development-Iraq \\ karzanwakil@gmail.com
}

\begin{abstract}
A Robin problem is a mixed problem with a linear combination of Dirichlet and Neumann D-N conditions. The aim of this paper are presents a new boundary integral equation BIE method for the solution of unbounded Robin boundary value problem $B V P$ in the simply connected domain. The method show how to reformulate the Robin boundary value problem BVP as Riemann-Hilbert problem RHP which lead to the system of integral equation, and the related differential equations are also created that give rise to unique solutions. Numerical results on several tests regions by the Nyström method NM with the trapezoidal rule TR are presented to clarify the solution technique for the Robin problem when the boundaries are sufficiently smooth.
\end{abstract}

Keywords: Robin problem, Riemann-Hilbert problem, Integral equation, Generalized Neumann kernel, Simply connected region.

\section{INTRODUCTION}

Many applications of the Laplacian differential operator are related to physical geodesy, measurement [1-2], while the application of mixed boundary value problem has been developed only during recent century [3]. In this study the mixed boundary value problem in the literature is the mixed D-N boundary value problem BVP. In this paper the applications of the mixed D-N of BVP in potential theory can be seen in [4]. A mixed BVP has mixed D-N type boundary conditions [5-6].

A Robin problem is a mixed BVP with a linear combination of D-N conditions, commonly called a Robin condition [6]. Many analytical methods for computing the Robin BVP for the Laplace's equation $\Delta \mathrm{u}=0$ in a simply connected region are limited to special domains. For general shape region, we have to resort to numerical methods [7-9]. Robin's condition is also called the third boundary condition in some books such as [10-12].

Robin problem was named after French mathematician called Victor Gustave Robin (1855-1897). He was a professor of applied mathematics, specifically in the field of mathematical physics at Sorbonne University in Paris [13]
At recent days, the interplay of RHP and integral equation with the generalized Neumann kernel GNK has been investigated in [14] for simply connected regions with smooth boundaries and in [15-16] for bounded and unbounded doubly connected and multiply connected regions.

In this paper we are compute the Robin BVP by reducing it to form of RHP, hence as long as the related system of boundary integral equation BIE method. By the additional conditions to the Robin BVP are given to obtain the unique solution.

\section{NOTATIONS AND AUXILIARY MATERIAL}

Consider the unbounded Robin problem on an arbitrary simply connected domain $\Omega$ with the boundary $\Gamma$. It consists of finding a function $u$ harmonic in the domain, continuous on domain and the boundary and satisfies the Robin's equation. An unbounded simply connected region with clockwise orientation boundary $\Gamma$ which is a smooth Jordan curve (see Figure 1).

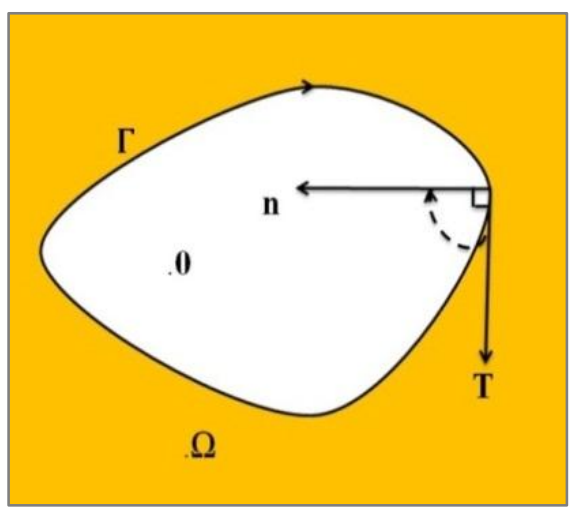

Figure 1 An unbounded simply connected domain $\Omega$.

The curve $\Gamma$ is parameterized by $2 \pi$-periodic twice continuously differentiable complex function $\eta(t)$ with non-vanishing first derivative.

In this paper, our contribution to the Robin BVP for choosing a harmonic function $u$, harmonic in domain $\Omega$, and continuous on domain and the boundary and satisfies the Robin's equation [10]. 
$\alpha(t) u(\eta(t))+\beta(t) \frac{\partial u(\eta(t))}{\partial \mathbf{n}}=l(t) \quad \alpha(t), \beta(t) \neq 0, \eta(t) \in \Gamma$,

where $\mathbf{n}$ is exterior normal vector to $\Gamma$. If $\frac{\alpha(t)}{\beta(t)}>0$, then the Robin BVP has a uniquely solution (see e.g [11, p. 163] and [12, p. 141]).The RHP can be computed a BIE with the GNK. This paper, relate the Robin problem with the RHP. We are define the real kernels in GNK and as imaginary kernel in GNK and real parts in GNK [8-9].

The RHP is consists of finding a function $g$ is analytic in the domain $\Omega$.

$$
\operatorname{Re}[A g]=\gamma
$$

Theorem 1. ([8-13]) If $g$ in the RHP equation $\operatorname{Re}[A g]=\gamma$ is a solution of the RHP with BVP

$$
A g=\gamma+\mathrm{i} \mu,
$$

Then from equation (2) the imaginary part $\mu$ is satisfies the BIE

$$
\mu-\mathbf{N} \mu=-\mathrm{M} \gamma .
$$

In this work the solubility of BIE with the GNK is specific by the index of the peroidic function $A(t)$ [9].

Theorem 2. ([14] Cauchy Integral Formula CIF) Let $f$ be a function that is analytic everywhere in $\Omega$ and on a simple closed contour $\Gamma$. If $\mathrm{z}$ is any point in side the domain $\Omega$. Then the CIF is

$\frac{1}{2 \pi i} \int_{\Gamma} \frac{f(\eta)}{\eta-z} d \eta=\left\{\begin{array}{cc}f(z)-f(\infty), & \mathrm{z} \in \Omega, \\ -f(\infty), & \text { For unbounded } \\ & \mathrm{z} \notin \Omega .\end{array}\right.$

\section{METHODOLOGY OF ROBIN PROBLEM}

We consider the methodology of computingthe Robin BVP with the Robin condition in an unbounded simplyconnected region as shown in Figure 2.

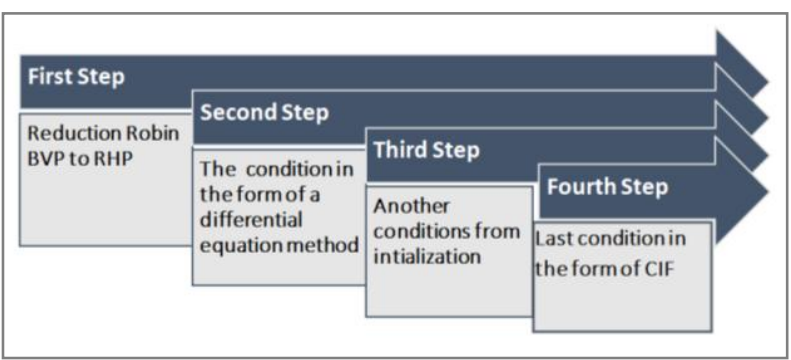

Figure2 Flowchart of Methodology

It can be shown that the Robinproblem (1) cab be reformulated as [15-16]:

$$
\begin{aligned}
& A(t) g(\eta(t))=\int_{0}^{t} \frac{l(\tau)|\dot{\eta}(\tau)| \cos \zeta(\tau)}{\beta(\tau)} d \tau+\mathrm{i} \int_{0}^{t} \frac{l(\tau)|\dot{\eta}(\tau)| \sin \zeta(\tau)}{\beta(\tau)} d \tau \\
& +\mathrm{i} \int_{0}^{t} \frac{\mu(\tau) \cos \zeta(\tau)}{\beta(\tau)} d \tau-\int_{0}^{t} \frac{\mu(\tau) \sin \zeta(\tau)}{\beta(\tau)} d \tau+c_{1}+\mathrm{i} c_{2} \\
& =\gamma_{1}(t)+\mathrm{i} \gamma_{2}(t)+\mathrm{i} \mu_{1}(t)-\mu_{2}(t)+c_{1}+\mathrm{i} c_{2} \\
& =\left(\gamma_{1}(t)-\mu_{2}(t)+c_{1}\right)+\mathrm{i}\left(\gamma_{2}(t)+\mu_{1}(t)+c_{2}\right), \quad t \in J,
\end{aligned}
$$

Where $c_{1}, c_{2}$, are unknown real constants in $H$, and

$$
\begin{array}{ll}
\gamma_{1}(t):=\int_{0}^{t} \frac{l(\tau)|\dot{\eta}(\tau)| \cos \zeta(\tau)}{\beta(\tau)} d \tau, & t \in J, \\
\gamma_{2}(t):=\int_{0}^{t} \frac{l(\tau)|\dot{\eta}(\tau)| \sin \zeta(\tau)}{\beta(\tau)} d \tau, & t \in J,
\end{array}
$$

Are known functions in $H$, and

$$
\begin{array}{ll}
\mu_{1}(t):=\int_{0}^{t} \frac{\mu(\tau) \cos \zeta(\tau)}{\beta(\tau)} d \tau, & t \in J, \\
\mu_{2}(t):=\int_{0}^{t} \frac{\mu(\tau) \sin \zeta(\tau)}{\beta(\tau)} d \tau, & t \in J,
\end{array}
$$

Are unknown functions in $H$ [15-16].Then the equation (5) can be written briefly describe as equation (5) where

$$
\begin{aligned}
& \gamma(t)=\gamma_{1}(t)-\mu_{2}(t)+c_{1} \\
& \mu(t)=\gamma_{2}(t)+\mu_{1}(t)+c_{2}
\end{aligned}
$$

From the equation (5) the real part produce the RHP. The periodic function $A(t)=e^{\mathrm{i} \zeta(t)}$ is not periodic in general.To stratify the result of Theorem 1, the periodic function $A(t)$ must be satisfy the condition $\zeta(2 \pi)-\zeta(0)=2 \pi$. Then by the Theorem 1 implies that equation(2) becomes the integral equation

$$
(\mathbf{I}-\mathbf{N})\left(\gamma_{2}(s)+\mu_{1}(s)+c_{2}\right)=-\mathbf{M}\left(\gamma_{1}(s)-\mu_{2}(s)+c_{1}\right) .
$$

Then applying both integral operators $\mathbf{N}$ and $\mathbf{M}[8,9]$, we obtain

$$
\begin{aligned}
& \mu_{1}(s)-\int_{J} N(\eta(s), \eta(t)) \mu_{1}(t) d t-\int_{J} M(\eta(s), \eta(t)) \mu_{2}(t) d t \\
& +\int_{J} M(\eta(s), \eta(t)) c_{1} d t+\left(c_{2}-\int_{J} N(\eta(s), \eta(t)) c_{2} d t\right. \\
& =-\int_{J} M(\eta(s), \eta(t)) \gamma_{1}(t) d t-\left(\gamma_{2}(s)-\int_{J} N(\eta(s), \eta(t)) \gamma_{2}(t) d t\right),
\end{aligned}
$$

The system of integral equations (13) is in two unknown real functions $\mu_{1}(t), \mu_{2}(t)$ also two unknown real constants $c_{1}, c_{2}$. In this work by both functions $\mu_{1}(t)$ $\mu_{2}(t)$ are given in equations (7),(8) respectivly, have a condition in the form of a differential equation

$$
\sin (\zeta(t)) \mu_{1}^{\prime}(t)-\cos (\zeta(t)) \mu_{2}^{\prime}(t)=0, \quad t \in J,
$$

And the conditions 


$$
\mu_{1}(0)=0, \quad \text { and } \quad \mu_{2}(0)=0 .
$$

For the exterior Robin problem, we have $f(\infty)=0$ which implise $g(\infty)=0$. Then applying the CIF for unbounded domain $\Omega$ in the Theorem 2, we have together with (5), (10) and (11), yields

$$
\begin{aligned}
& g(\infty)=\frac{1}{2 \pi \mathrm{i}} \int_{\Gamma} \frac{g(\eta)}{\eta} d \eta=\frac{1}{2 \pi} \int_{\Gamma} \frac{g(\eta(t))}{\eta(t)} \dot{\eta}(t) d t \\
& =\frac{1}{2 \pi \mathrm{i}} \int_{0}^{2 \pi} \frac{\left[\gamma_{1}(t)-\mu_{2}(t)+c_{1}+\mathrm{i}\left(\gamma_{2}(t)+\mu_{1}(t)+c_{2}\right)\right] \dot{\eta}(t)}{A(t) \eta(t)} d t, \quad t \in J .
\end{aligned}
$$

Thus $g(\infty)=0$, implies that

$\frac{1}{2 \pi \mathrm{i}} \int_{0}^{2 \pi}[\gamma(t)+\mathrm{i} \mu(t)]\left[\operatorname{Re} \frac{\dot{\eta}(t)}{A(t) \eta(t)}+\mathrm{i} \operatorname{Im} \frac{\dot{\eta}(t)}{A(t) \eta(t)}\right] d t=0$.

The real part and imagenary part of equation (17) gives more conditions as

$\int_{0}^{2 \pi} \operatorname{Re} \frac{\dot{\eta}(t)}{A(t) \eta(t)} \mu_{1}(t) d t-$

$\int_{0}^{2 \pi} \operatorname{Im} \frac{\dot{\eta}(t)}{A(t) \eta(t)} \mu_{2}(t) d t+c_{1} \int_{0}^{2 \pi} \operatorname{Im} \frac{\dot{\eta}(t)}{A(t) \eta(t)} d t+$

$c_{2} \int_{0}^{2 \pi} \operatorname{Re} \frac{\dot{\eta}(t)}{A(t) \eta(t)} d t=-\int_{0}^{2 \pi} \operatorname{Re} \frac{\dot{\eta}(t)}{A(t) \eta(t)} \gamma_{2}(t) d t-$

$\int_{0}^{2 \pi} \operatorname{Im} \frac{\dot{\eta}(t)}{A(t) \eta(t)} \gamma_{1}(t) d t$,

and

$-\int_{0}^{2 \pi} \operatorname{Im} \frac{\dot{\eta}(t)}{A(t) \eta(t)} \mu_{1}(t) d t-$

$\int_{0}^{2 \pi} \operatorname{Re} \frac{\dot{\eta}(t)}{A(t) \eta(t)} \mu_{2}(t) d t+c_{1} \int_{0}^{2 \pi} \operatorname{Re} \frac{\dot{\eta}(t)}{A(t) \eta(t)} d t-$

$c_{2} \int_{0}^{2 \pi} \operatorname{Im} \frac{\dot{\eta}(t)}{A(t) \eta(t)} d t=\int_{0}^{2 \pi} \operatorname{Im} \frac{\dot{\eta}(t)}{A(t) \eta(t)} \gamma_{2}(t) d t-$

$\int_{0}^{2 \pi} \operatorname{Re} \frac{\dot{\eta}(t)}{A(t) \eta(t)} \gamma_{1}(t) d t$,

Finally to compute the Robin BVP (1) on exterior domain, we compute for $\mu_{1}(t), \mu_{2}(t), c_{1}$ and $c_{2}$ from (14),(14), (15), (18) and (19). Then we compute $g(\eta(t))$ from (5).Furthermore, by the relationship of $g=-i f$ we get $f(\eta(t))=\mathrm{i} g(\eta(t))$ and hence $u(\eta(t))=$ $\operatorname{Re}[g(\eta(t))]$. The interior values of $f(\eta(t))$ can be calculate with the CIF (4) which yields $u(z)=$ $\operatorname{Re}[g(z)]$, when the values $\mathrm{z}$ in the exterior domain $\Omega$.

\section{IMPLEMENTATION}

The functions in the form of both $A(t)$ and $\eta(t)$ are $2 \pi$-periodicfunction respectively, then the integral equation in (12) can be better discretized in this study on an equidistant connection by the Nystrom method NM with the Trapezoidal rule TR by using $n$ equidistant nodes [17]. Since $\mathbf{M}=\mathbf{M}_{\mathbf{1}}-\mathbf{K}$, in this work the singular kernel $\mathbf{K}$ are discretized using the Wittich's method WM [18]. Hence, now we have $n$ equations in $2 n+2$ unknowns variables

$\mu_{1}\left(t_{1}\right), \mu_{1}\left(t_{2}\right), \ldots, \mu_{1}\left(t_{n}\right), \mu_{2}\left(t_{1}\right), \mu_{2}\left(t_{2}\right), \ldots, \mu_{2}\left(t_{n}\right), c_{1}, c_{2}$.

This equation (14) will be discretized to obtain $n$ more equations [19]. We now have $2 n$ equations in $2 n+2$ in unknowns variables (20). For the Robin BVP on unbounded simply connected domain, we also have two conditionsfrom (15), we obtained $(2 n+2)$ by $(2 n+2)$. Finally, the two more equations from (18) and (19) are respectively add to give a $(2 n+4)$ by $(2 n+2)$ linear system.

In this case, the obtained linear systems are computed by using the MATLAB softwere (2011a), 32-bit operating system.

From the computed solutions

$\mu_{1}(t), \mu_{2}(t), \gamma_{1}(t), \gamma_{2}(t), c_{1}$ and $c_{2}$, the approximate boundary values of the analytic function $f_{n}(\eta(t))$ are calculated using the formula

$$
f_{n}(\eta(t))=\frac{-\left(\gamma_{2}(t)+\mu_{1}(t)+c_{2}\right)+\mathrm{i}\left(\gamma_{1}(t)-\mu_{2}(t)+c_{1}\right)}{e^{\mathrm{i} \zeta(t)}} .
$$

We approximate interior values of the equation (21) are calculated with the CIF in the form of

$$
f(z)=\frac{f(\infty)+\frac{1}{2 \pi \mathrm{i}} \int_{J} \frac{f(\eta)}{\eta-z} d \eta}{1+\frac{1}{2 \pi \mathrm{i}} \int_{J} \frac{d \eta}{\eta-z}} .
$$

Here $f(\infty)=0$, then approximate the integral in (22) for exterior region [20] then computed by using the trapezoidal rule.

\section{NUMERICAL RESULT AND EXAMPLE}

Considered two examples computing of Robin BVP in exterior domain.

Example1. In this examplewe coonsider the domain $\Omega$ are bounded by

$$
\Gamma: \eta(t)=e^{-i t}, \quad 0 \leq t \leq 2 \pi .
$$

For $\alpha(t), \beta(t), u(t)$ in (1), we choose

$$
\alpha(t)=1-0.2 \sin t, \text { and } \quad \beta(t)=1 .
$$

Thefunction $l(t)=2 \cos t+0.5 \cos ^{2} t$ in (1) the exact solution in this example is $f(z)=\frac{1}{z}$, 
The real constantes are $c_{l}=0$ and $c_{2}=-1$. For this example, $A(t)=e^{i(t+0.5 \sin t)}, t \in J=[0,2 \pi]$.

Table 1 and Table 2 are lists the maximum error norm $\left\|u(\eta(t))-u_{n}(\eta(t))\right\|_{\infty}$ and the error $\left\|f(z)-f_{n}(z)\right\|$ respectively are plotted in Figure 3 and Figure 4 as shown below with $n=512$.

Table 1: The error $\left\|u(\eta(t))-u_{n}(\eta(t))\right\|_{\infty}$ for Example 1 .

\begin{tabular}{|l|r|}
\hline $\boldsymbol{n}$ & $\left\|\boldsymbol{u}(\boldsymbol{\eta}(\boldsymbol{t}))-\boldsymbol{u}_{\boldsymbol{n}}(\boldsymbol{\eta}(\boldsymbol{t}))\right\|_{\infty}$ \\
\hline 32 & $9.20(-3)$ \\
\hline 64 & $3.87(-4)$ \\
\hline 128 & $2.66(-5)$ \\
256 & $1.66(-6)$ \\
512 & $1.04(-7)$ \\
1024 & $6.50(-9)$ \\
\hline
\end{tabular}

Table 2: Absolute error $\left|f(z)-f_{n}(z)\right|$ at some selected points on domain $\Omega$.

\begin{tabular}{|l|c|c|c|}
\hline $\boldsymbol{n}$ & $\mathbf{- 1 . 3 + 1 . 1 i}$ & $\mathbf{- 1 . 2 + 1 . 2 i}$ & $\mathbf{- 1 - 1 . 5 i}$ \\
\hline 32 & $5.49(-5)$ & $7.36(-5)$ & $7.85(-5)$ \\
\hline 64 & $6.15(-6)$ & $7.29(-6)$ & $8.34(-6)$ \\
\hline 128 & $4.75(-7)$ & $5.53(-7)$ & $6.16(-7)$ \\
256 & $3.16(-8)$ & $3.66(-8)$ & $4.06(-8)$ \\
512 & $2.02(-9)$ & $2.33(-9)$ & $2.59(-9)$ \\
\hline
\end{tabular}



Figure 3 The error $\left|u(z)-u_{n}(z)\right|$ for Example 1 with $n=512$.

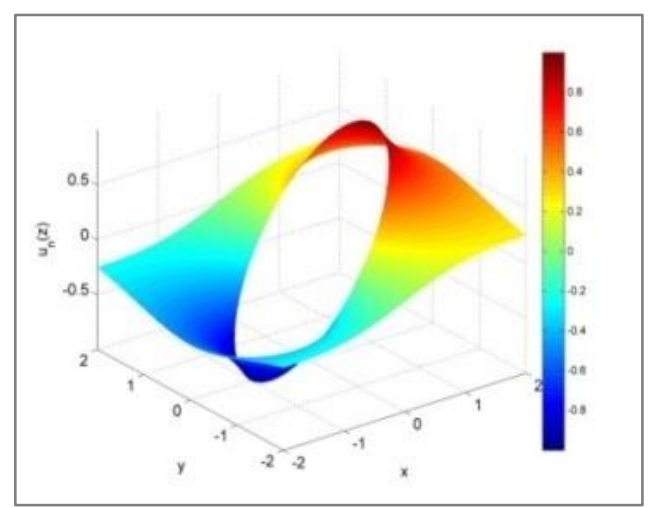

Figure 4 The approximation solution $u_{n}(z)$ for Example 1 with $n=512$.

Example2. In this example also we coonsider the domain $\Omega$ are bounded by
$\Gamma: \eta(t)=(1.8+0.5 \cos t) e^{-i t}$
$0 \leq t \leq 2 \pi$

In (1) the exact solution of this example is $f(z)=\frac{1}{z}$,

The real constants are $c_{1}=0, c_{2}=-0.4348$.

Table 3 and Table 4 are lists the maximum error norm $\left\|u(\eta(t))-u_{n}(\eta(t))\right\|_{\infty}$ and the error $\left\|f(z)-f_{n}(z)\right\|$ respectively are plotted in Figure 5 and Figure 6 as shown below with $n=512$.

Table 3: The error $\left\|u(\eta(t))-u_{n}(\eta(t))\right\|_{\infty}$ for Example 2.

\begin{tabular}{|l|c|}
\hline $\boldsymbol{n}$ & $\begin{array}{c}\| \boldsymbol{u}(\boldsymbol{\eta}(\boldsymbol{t})) \\
-\boldsymbol{u}_{\boldsymbol{n}}(\boldsymbol{\eta}(\boldsymbol{t})) \|_{\infty}\end{array}$ \\
\hline 32 & $9.45(-3)$ \\
\hline 64 & $4.29(-4)$ \\
\hline 128 & $1.84(-5)$ \\
256 & $1.32(-7)$ \\
512 & $1.01(-8)$ \\
\hline
\end{tabular}

Table 4: Absolute error $\left|f(z)-f_{n}(z)\right|$ at some selected points on domain $\Omega$.

\begin{tabular}{|l|c|c|c|c|}
\hline $\boldsymbol{n}$ & $\mathbf{1 . 1 + 1 . 1 i}$ & $\mathbf{- 1 . 1 - 1 . 1 i}$ & $\mathbf{1 . 1 - 1 . 1 i}$ & $\mathbf{- 1 . 1 + 1 . 1 i}$ \\
\hline 32 & $2.70(-3)$ & $2.10(-3)$ & $1.40(-3)$ & $1.80(-3)$ \\
\hline 64 & $1.12(-4)$ & $9.63(-5)$ & $1.07(-4)$ & $9.41(-5)$ \\
\hline 128 & $7.06(-6)$ & $6.00(-6)$ & $6.94(-6)$ & $5.92(-6)$ \\
256 & $4.40(-7)$ & $3.75(-7)$ & $4.34(-7)$ & $3.69(-7)$ \\
512 & $4.77(-8)$ & $2.34(-8)$ & $2.71(-8)$ & $2.30(-8)$ \\
1024 & $1.72(-9)$ & $1.40(-9)$ & $1.69(-9)$ & $1.43(-9)$ \\
\hline
\end{tabular}

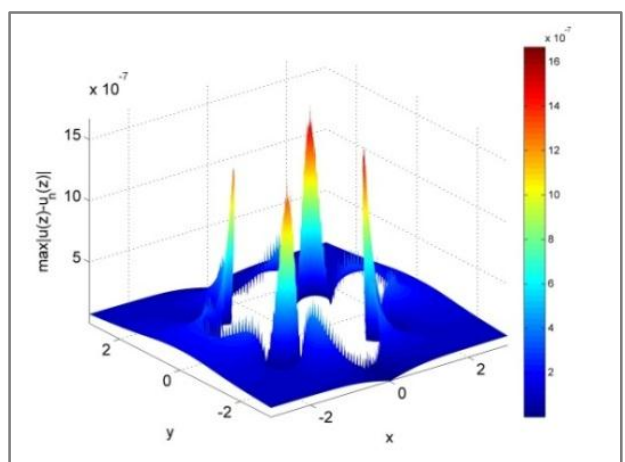

Figure 5 The error $\left|u(z)-u_{n}(z)\right|$ for Example 2 with $n=512$.

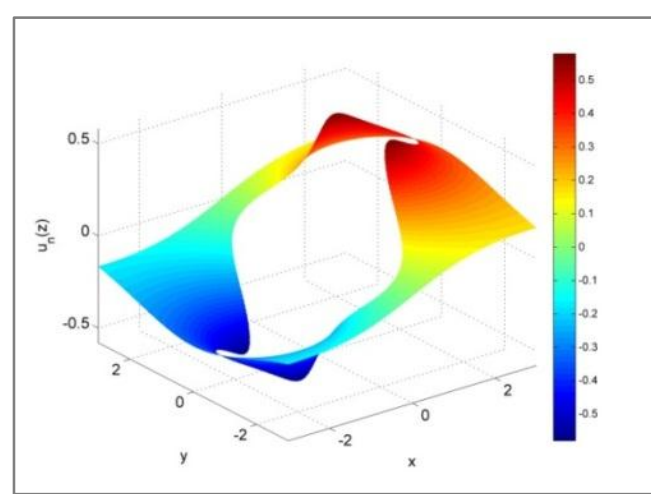

Figure 6 The approximation solution $u_{n}(z)$ for Example 2 with $n=512$.

\section{CONCLUSIONS}

We have solved exterior Robin problems in simply connected domain with smooth boundaries using a 
combination of a system of integral equation and the differential equations. In this paper, the integral equation is discretized by the Nystrom method NM with the Trapezoidal rule TR and the Wittich's method WM in [15-16], however in this study the differential equation is discretized in [19]. In conclusion, the presented numerical results clarify that our method can be used to make approximations [20] of good accuracy and best results.

\section{REFERENCES}

[1] W. Heiskanen,H. Moritz. Physical Geodesy. San Francisco / London. 1967

[2] W. Fang, Z. Suxing. Numerical recovery of Robin boundary from boundary measurements for the Laplace equation.Journal of Computational and Applied Mathematics, 224:573-580, 2009.

[3] K. Gustafson, A. Takehisa. The third boundary condition was it Robin's?The Mathematical Intelligencer, 20:63-71, 1998.

[4] I. N. Sneddon.Mixed Boundary Value Problems In Potential Theory. North-Holland. 1966

[5] M. M. S. Nasser, A. H. M. Murid, M. Ismail, E. M. A. Alejaily. Boundary integral equations with the generalized Neumann kernel for Laplace's equation in multiply connected regions. Appl. Math. Comput, 217:4710-4727, 2011.

[6] S. A. A. Alhatemi, A. H. M. Murid, M. M. S. Nasser. A boundary integral equation with the generalized Neumann kernel for a mixed boundary value problem in unbounded multiply connected regions.Boundary Value Problems, 1: 1-17, 2013.

[7] M. M. S. Nasser. Numerical conformal mapping via a boundary integral equation with the generalized Neumann kernel. SIAM Journal on Scientific Computing, 31:1695-1715, 2009.

[8] R. Wegmann, A. H. M. Murid, M. M. S. Nasser. The Riemann-Hilbert problem and the generalized Neumann kernel.Journal of Computational and Applied Mathematics, 182:388-415, 2005.

[9] R. Wegmann, M. M. S. Nasser.The RiemannHilbert problem and the generalized Neumann kernel on multiply connected regions.Journal of Computational and Applied Mathematics, 214:3657, 2008.

[10] T. Petrila. Complex value boundary element method for some mixed boundary value problems. Studia Univ, Babes-Bolyai, Informatica, 44:37-42, 1999.

[11] R. M. M. Mattheij, S. W Rienstra, J. H. M. ten Thije Boonkkamp. Partial Differential Equations Modeling, Analysis, Computation.SIAM. 2005

[12]S. Salsa.Partial Differential Equations in Action From Modeling to Theory.Springer Science and Business Media. 2008
[13] M. M. S. Nasser. The Riemann-Hilbert problem and the generalized Neumann kernel on unbounded multiply connected regions. The University Researcher Journal, 20: 47-60, 2009.

[14]F. D. Gakhov. Boundary Value Problem. Oxford Pergamon Press. 1966

[15] S. H. H. Al-Shatri, A. H. M. Murid, M. Ismail, M. I. Muminov. Solving Robin problems in multiply connected regions via an integral equation with the generalized Neumann kernel.Boundary Value Problem.2016, 1-23,2016.

[16] S. H. H. Al-Shatri, A. H. M. Murid, M. Ismail. Solving Robin problems in bounded doubly connected regions via an integral equation with the generalized Neumann kernel. AIP Conference Proceedings.1750,1, p. 030004) 2016.

[17] K. E. Atkinson. The Numerical Solution of Integral Equations of the Second Kind. Cambridge: Cambridge University Press. 1997

[18]D. Gaier. Konstruktive Methoden der Konformen Abbildung.Berlin-Göttingen-Heidelberg. 1964

[19] M. Abramowitz, I. E. Stegun. Handbook of Mathematical Functions, with Formulas, Graphs, and Mathematical Tables. Courier Corporation. 1964

[20] J. Helsing, R. Ojala. On the evaluation of layer potentials close to their sources. J. Comput. Phys, 227:2899-2921, 2008. 